\begin{tabular}{|c|c|c|}
\hline \multirow{3}{*}{$\begin{array}{r}\text { Case Reports in } \\
\text { Gastroenterology }\end{array}$} & \multicolumn{2}{|c|}{ Case Rep Gastroenterol 2016;10:653-660 } \\
\hline & $\begin{array}{l}\text { DOI: } 10.1159 / 000452203 \\
\text { Publisnea oninne. TNovember 7, } 2016\end{array}$ & $\begin{array}{l}\text { ( ) } 2016 \text { The Author(s) } \\
\text { Published by S. Karger AG, Basel } \\
\text { www.karger.com/crg }\end{array}$ \\
\hline & $\begin{array}{l}\text { This article is licensed under the } \\
\text { International License (CC BY-NC) } \\
\text { Usage and distribution for commercia }\end{array}$ & $\begin{array}{l}\text { nons Attribution-NonCommercial } 4.0 \\
\text { ger.com/Services/OpenAccessLicense). } \\
\text { uires written permission. }\end{array}$ \\
\hline
\end{tabular}

\title{
Disseminated Histoplasmosis: A Challenging Differential Diagnostic Consideration for Suspected Malignant Lesions in the Digestive Tract
}

\author{
Bernhard Doleschal $^{\mathrm{a}} \quad$ Therese Rödhammer $^{\mathrm{b}} \quad$ Oleksiy Tsybrovskyy $^{\mathrm{c}}$ \\ Karl J. Aichberger ${ }^{a}$ Franz Lang ${ }^{a}$ \\ ${ }^{a}$ Department of Internal Medicine I, Hematology, Oncology and Gastroenterology, \\ Hospital of the Sisters of Charity, Linz, Austria; ${ }^{b}$ Department of Otorhinolaryngology, \\ Hospital of the Sisters of Charity, Linz, Austria; ${ }^{\mathrm{C}}$ Department of Pathology, Hospital of the \\ Sisters of Charity, Linz, Austria
}

\section{Keywords}

Disseminated histoplasmosis - Nonimmunocompromised patient · Oral ulcers · Colonic lesions $\cdot$ Nonendemic area $\cdot$ Systemic illness

\begin{abstract}
Histoplasmosis is well characterized as an endemic fungal disease restricted to certain areas of the USA. In Middle Europe, most patients present with acute pulmonary symptoms after travelling to endemic areas. Here, we want to illustrate the case of a 67-year-old man who presented with persistent oral ulcers, hoarseness, dysphagia, diarrhea, and weight loss to our Department of Otorhinolaryngology in December 2014. He was a retired construction worker and had a history of soil-disruptive activities in Africa and Middle and South America during employment. A positron emission tomography-computed tomography scan revealed prominent hypermetabolic lesions in the cecum and the lung, pointing towards a malignant disease. Surprisingly, histological examination of colonic and oral biopsies revealed abundant intracellular fungal elements, highly suspicious of Histoplasma capsulatum. Diagnosis was finally confirmed by panfungal polymerase chain reaction. Upon treatment with liposomal
\end{abstract}


amphotericin followed by itraconazole, the severely ill patient showed an impressive clinical response. This case describes a disseminated manifestation of $H$. capsulatum years after the first exposure in an otherwise immunocompetent patient descending from a nonendemic area.

(c) 2016 The Author(s)

Published by S. Karger AG, Basel

\section{Introduction}

Histoplasma capsulatum - first described by Dr. Samuel Darling [1] in 1906 - is a dimorphic fungal organism found in moist fertile soil contaminated by bird or bat droppings. Although endemic in East and Central US states bordering the Ohio River valley and the lower Mississippi River, in Europe some rare autochthone cases in Italy, near the Po valley, have been described. However, most cases reported in Europe seem to be imported [2].

Transmission regularly occurs via inhalation of $H$. capsulatum spores from the soil, and primary infections in the immunocompetent host are generally asymptomatic or present as a self-limiting flu-like illness. If infection cannot be cleared, as in immunocompromised patients with disabled cellular immunity, the organism continues to reproduce intracellularly and disseminates throughout the body via lymphatic and hematogenous circulation, cumulating in a state called disseminated histoplasmosis. Disseminated histoplasmosis manifests most prominently in the digestive tract, from mouth to anus, beside its well-described symptoms and manifestations, such as pulmonary disease, skin lesions, fever, and encephalopathy [3]. Untreated disseminated histoplasmosis usually leads to death within a few weeks. Therefore, accurate diagnosis and appropriate treatment are absolutely mandatory.

Here, we present the case of an obviously nonimmunocompromised patient from a nonendemic area infected by $H$. capsulatum, and we thus describe the first case with a disseminated disease course in Austria.

\section{Case Presentation}

A 67-year-old man was referred to our Department of Otorhinolaryngology in December 2014 for the evaluation of anorexia, painful dysphagia, hoarseness, diarrhea, and a history of unintentional weight loss of about $15 \mathrm{~kg}$ (equaling approximately $20 \%$ of his previous body weight of roughly $80 \mathrm{~kg}$ ) in the last 3 months, accompanied by intermittent fever, night sweats, and chills. His past medical history consisted of stable coronary heart disease and well-controlled chronic obstructive pulmonary disease caused by long-term tobacco smoking (about 30 pack-years). His long-term medication consisted of aspirin and inhaled anticholinergic and corticosteroid bronchodilators.

He was a retired engineer employed at a local steel company, operative in Africa and Middle and South America, where the patient was involved in construction work and soildisruptive activities for several months. However, there was no history of far distance travelling during the last 6 years.

On admission, the patient was in an alarming general condition, cachectic with a wasting syndrome, requiring intermittent parenteral nutrition. The most obvious clinical observation was an extremely painful stomatitis and angular cheilitis with ulcers involving the tongue and the palate (Fig. 1a, b). Laboratory data on admission included an unremarkable blood count with slightly elevated C-reactive protein of $3.8 \mathrm{mg} / \mathrm{dL}$, an increased lactate dehydrogenase of $264 \mathrm{U} / \mathrm{L}$, and a mildly elevated angiotensin-converting enzyme level of 64 
$\mathrm{U} / \mathrm{L}$ in the serum. Screening for tumor markers and autoimmune disease as well as testing results for HIV, hepatitis, and tuberculosis were negative. No evidence of immunodeficiency could finally be provided. Chest X-ray demonstrated an emphysematous lung with reticulonodular parenchymal changes (Fig. 2a).

Further evaluation for a suspected occult malignant disease, bearing in mind the longterm tobacco consumption, included a whole-body positron emission tomography scan, which detected an additional hypermetabolic process in the ascending colon (Fig. 2b). This prompted us to perform an endoscopic evaluation of the colon and the terminal ileum. Macroscopically, rather small ulcerous lesions and erosions presented on a diffuse edematous mucosa with capillary congestion in the terminal ileum and ascending colon upon endoscopic examination (Fig. 2c). Histological examination of the biopsies excluded a malignant process but rather described a polypoid mucosa infiltrated by a kind of granulomatous inflammatory process with the presence of prominent histiocytes. Intracellularly, these "foam cells" harbored abundant intracellular round-shaped fungal elements with a characteristic halo sign, about 2-4 $\mu \mathrm{m}$ in diameter, highly indicative of $H$. capsulatum (Fig. $2 \mathrm{~d}$ ).

Biopsies of the tongue and a computed tomography-guided puncture of the lung confirmed the presence of round intracellular fungal forms using periodic acid-Schiff staining (Fig. 1c, d). Diagnosis was finally established from an oral re-biopsy by panfungal polymerase chain reaction, performed at a reference center at the Medical University of Vienna, confirming positivity for H. capsulatum.

Liposomal amphotericin B (1 mg/kg body weight) was administered to the patient, and constitutional symptoms markedly improved during the next few days. Lesions in the oral cavity showed a dramatic positive response to antifungal treatment. After 4 weeks of treatment, the patient was discharged from hospital and switched to oral itraconazole.

On follow-up 6 months later (Fig. 3), the patient continued to improve and subjectively reported that his voice was much stronger and that his dysphagia and gastrointestinal symptoms had completely resolved.

\section{Discussion}

Disseminated histoplasmosis is tightly connected with a dysfunctional immune system. Particularly, conditions with compromised cellular immunity affecting $\mathrm{T}$ cells are prone to propagation of the disease, a scenario often resembling miliary tuberculosis [4]. Only a small proportion of exposed individuals $(<0.1 \%)$ may develop disseminated disease. AIDS, HTLV1 , hepatitis $\mathrm{C}$, renal failure, long-term corticosteroid use or therapy with biologicals, and older age [5] are well-characterized risk factors. Notably, up to $40 \%$ of patients presenting with disseminated histoplasmosis do not have any obvious risk factors [6].

Endogenous reactivation of old foci during an immunocompromised state might be the trigger of dissemination [7]. In our patient, the underlying chronic obstructive pulmonary disease might have been a risk factor for primary infection of the lung; however, we could not delineate any commonly acquired or inherited immune deficiency states often associated with disseminated histoplasmosis. Thus, reactivation years after the last stay in endemic areas, performing "soil-disruptive activities" [8], might just be attributed to an age-related immune system "rundown", an already previously described promoting factor for dissemination [5].

In clear contrast to the initial presentation of acute histoplasmosis, which almost exclusively involves the respiratory tract, disseminated histoplasmosis most prominently affects 
the gastrointestinal tract [4]. Typical sites with abundant lymphatic tissue, such as the terminal ileum, are mostly affected, presenting on endoscopic examination as segmental inflammation, ulcerative lesions, and also polypoid masses, often incorrectly attributed to malignancy, inflammatory bowel disease (e.g., Crohn's disease and ulcerative colitis), or appendicitis [9]. In the majority of patients, gastrointestinal manifestation takes a subclinical disease course. Nonetheless, endoscopy is an important diagnostic tool for the assessment of dissemination.

Dissemination can probably affect any organ, and this circumstance might contribute to a "chameleon-like" clinical picture and misleading diagnoses. Therefore, it appears crucial for clinicians to be aware that an oropharyngeal or laryngeal presentation of histoplasmosis may be the only apparent clinical sign of dissemination [10]. Patients suffer from hoarseness and dysphagia caused by indurated, ulcerated lesions mimicking cancer [11]. In addition, a differential diagnostic consideration should include tuberculosis, other fungal diseases, such as coccidioidomycosis and blastomycosis, and viral infection, such as coxsackievirus or herpes virus infection.

Microscopically, diffuse lymphohistiocytic infiltrates with fungal elements about 2-4 $\mu \mathrm{m}$ in size detected within the cytoplasm of macrophages are pathognomonic. Well-formed granulomas are rare, whereas lymphohistiocytic nodules are more often noted [9].

Granulomatous inflammatory processes are often associated with elevated angiotensinconverting enzyme serum levels, and previous reports even suggested exclusion of histoplasmosis, especially during acute disease states, before establishing the diagnosis of sarcoidosis [12].

Although serological testing for antibodies to $H$. capsulatum revealed a negative result in our case, this seems to be in accordance with previously published reports of diminished sensitivity of serological tests in disseminated disease, especially during immunocompromised states. Periodic acid-Schiff staining is considered the gold-standard technique for microscopically based diagnosis, but sensitivity might be hampered by sampling errors, lack of expertise, or the impossibility to obtain representative material from severely ill patients [13].

We employed a polymerase chain reaction to confirm the diagnosis. Although sensitivity of polymerase chain reaction is highly dependent on the type of isolate (culture vs. direct clinical), its impact lies in the reliable diagnostic differentiation between different fungal specimen [14].

The therapeutic approach to disseminated histoplasmosis is much more straightforward. Lipid formulation of amphotericin B at 3-5 mg/kg body weight per day, intravenously, for 1-2 weeks, followed by itraconazole at $200 \mathrm{mg} 3$ times daily for 3 days and then $200 \mathrm{mg}$ twice daily for a total of 12 weeks p.o., is the accepted gold-standard therapy for disseminated histoplasmosis [7]. Considering the severe illness of the patient, we decided for a prolongation of intravenous antifungal therapy up to 4 weeks. When clinical response was observed, antifungal therapy was replaced by oral itraconazole, and this treatment was continued for up to 1 year.

As disease monitoring by the detection of the $H$. capsulatum antigen was impossible due to its exclusive availability in the USA, response to therapy was monitored on a clinical basis, showing a dramatic symptomatic improvement. Macroscopically, the oral lesions completely vanished (Fig. 3). 


\section{Conclusion}

Disseminated histoplasmosis is an important differential diagnostic consideration in patients with systemic illness, especially when oropharyngeal and gastrointestinal lesions are dominating. The rising incidence of disseminated histoplasmosis in nonendemic areas emphasizes the need for a thorough examination of the patients' travelling history. Due to its heterogeneous clinical presentation, a close interaction between clinicians across the borders of their subdisciplines and between pathologists and microbiologists is mandatory for establishing the right diagnosis.

\section{Authors' Contribution}

B.D. wrote the manuscript. B.D., F.L., and T.R. participated in patient care and revised the manuscript. O.T. performed the pathology analysis and revised the manuscript. K.J.A. revised the manuscript.

\section{Funding Sources}

There were no funding sources.

\section{Statement of Ethics}

Informed consent was obtained from the patient.

\section{Disclosure Statement}

The authors declare that they have no conflicts of interest.

\section{References}

1 Darling ST: The Morphology of the parasite (Histoplasma capsulatum) and the lesions of histoplasmosis, a fatal disease of tropical America. J Exp Med 1909;11:515-531.

$\checkmark 2$ Ashbee HR, Evans EGV, Ashbee HR, Evans EGV, Viviani MA, Dupont B, et al: Histoplasmosis in Europe: report on an epidemiological survey from the European Confederation of Medical Mycology Working Group. Med Mycol 2008;46:57-65.

3 Wheat LJ: Histoplasmosis: a review for clinicians from non-endemic areas. Mycoses 2006;12:1-9.

-4 Kahi CJ, Wheat LJ, Allen SD, Sarosi GA: Gastrointestinal histoplasmosis. Am J Gastroenterol 2005;100:220-231.

-5 Wheat LJ, Slama TG, Norton JA, Kohler RB, Eitzen HE, French ML, et al: Risk factors for disseminated or fatal histoplasmosis. Analysis of a large urban outbreak. Ann Intern Med 1982;96:159-163.

-6 Assi MA, Sandid MS, Baddour LM, Roberts GD, Walker RC: Systemic histoplasmosis: a 15-year retrospective institutional review of 111 patients. Medicine (Baltimore) 2007;86:162-169.

7 Wheat LJ, Freifeld AG, Kleiman MB, Baddley JW, McKinsey DS, Loyd JE, et al: Clinical practice guidelines for the management of patients with histoplasmosis: 2007 update by the Infectious Diseases Society of America. Clin Infect Dis 2007;45:807-825.

-8 Huhn GD, Austin C, Carr M, Heyer D, Boudreau P, Gilbert G, et al: Two outbreaks of occupationally acquired histoplasmosis: more than workers at risk. Environ Health Perspect 2005;113:585-589. 


\section{Case Reports in \\ Gastroenterology}

\begin{tabular}{l|l}
\hline Case Rep Gastroenterol 2016;10:653-660 \\
\hline DOI: 10.1159/000452203 & $\begin{array}{l}\text { @ 2016 The Author(s). Published by S. Karger AG, Basel } \\
\text { www.karger.com/crg }\end{array}$ \\
\hline
\end{tabular}

Doleschal et al:: Disseminated Histoplasmosis: A Challenging Differential Diagnostic Consideration for Suspected Malignant Lesions in the Digestive Tract

-9 Zhu L-L, Wang J, Wang Z-J, Wang Y-P, Yang J-L: Intestinal histoplasmosis in immunocompetent adults. World J Gastroenterol 2016;22:4027-4033.

10 Goodwin RA, Shapiro JL, Thurman GH, Thurman SS, Prez Des RM: Disseminated histoplasmosis: clinical and pathologic correlations. Medicine (Baltimore) 1980;59:1-33.

11 Antonello VS, Zaltron VF, Vial M, Oliveira FM de, Severo LC: Oropharyngeal histoplasmosis: report of eleven cases and review of the literature. Rev Soc Bras Med Trop 2011;44:26-29.

$\rightarrow 12$ Ryder KW, Jay SJ, Kiblawi SO, Hull MT: Serum angiotensin converting enzyme activity in patients with histoplasmosis. JAMA 1983;249:1888-1889.

13 Hage CA, Azar MM, Bahr N, Loyd J, Wheat LJ: Histoplasmosis: up-to-date evidence-based approach to diagnosis and management. Semin Respir Crit Care Med 2015;36:729-745.

14 Babady NE, Buckwalter SP, Hall L, Le Febre KM, Binnicker MJ, Wengenack NL: Detection of Blastomyces dermatitidis and Histoplasma capsulatum from culture isolates and clinical specimens by use of realtime PCR. J Clin Microbiol 2011;49:3204-3208.
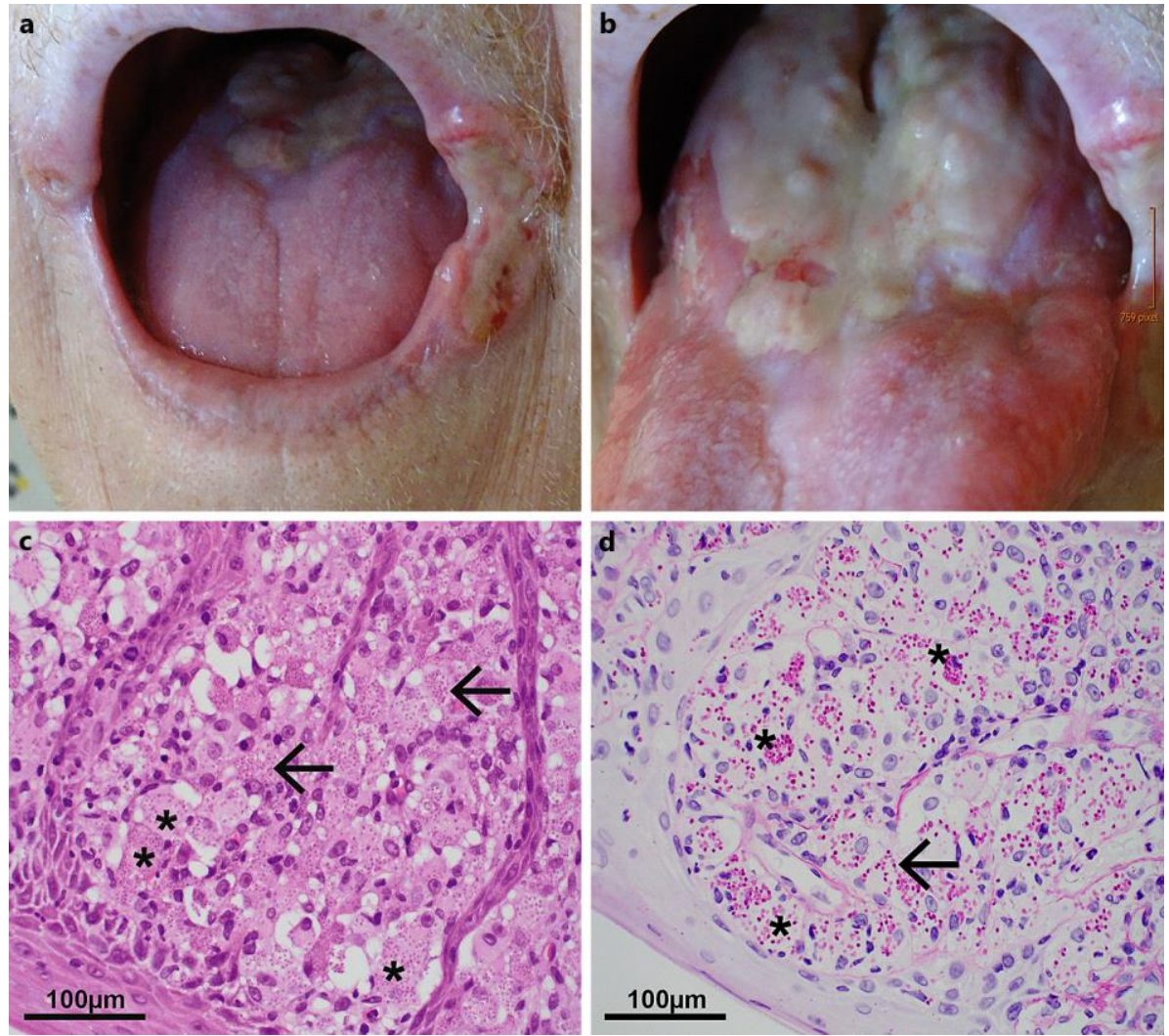

Fig. 1. a, b Fibrinous detritus-covered lesions of the tongue and angular cheilitis with irregular borders and hemorrhagic impingement in disseminated histoplasmosis clinically mimicking malignant disease (e.g., squamous cell carcinoma). c Biopsy taken from oral lesions showing diffuse inflammatory infiltration with abundant large histiocytes (indicated by asterisks), harboring multiple oval, pale eosinophilic bodies (indicated by arrows) with HE staining (40× magnification), morphologically suspicious of intracellular microorganisms. d Periodic acid-Schiff staining (40× magnification) of oral biopsy, highlighting round fungal organisms (indicated by an arrow), measuring about 2-3 $\mu \mathrm{m}$ in diameter, consistent with Histoplasma capsulatum. 

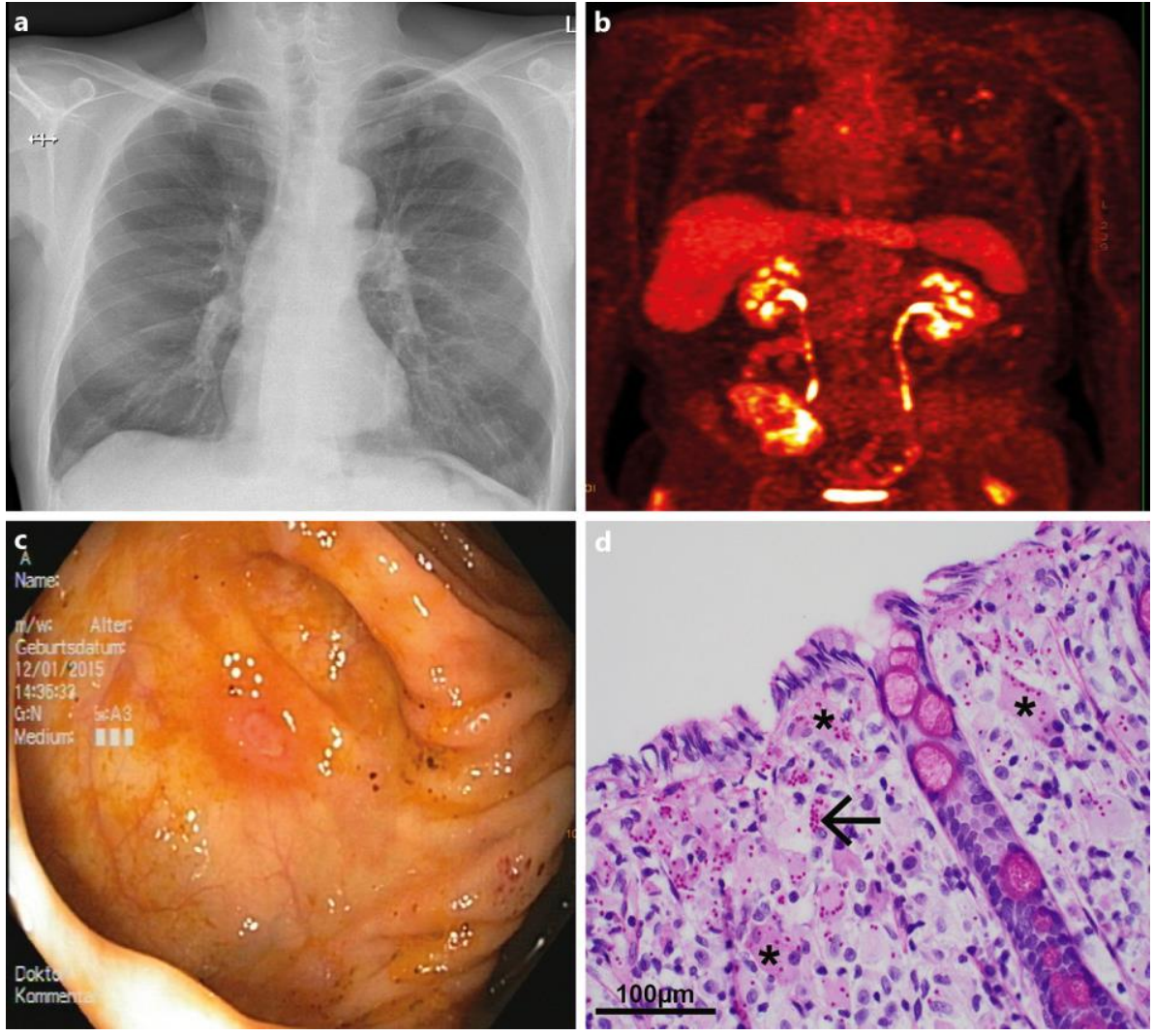

Fig. 2. a Chest X-ray demonstrating suspected malignant parenchymal cirrhotic changes. b Positron emission tomography-computed tomography revealing prominent hypermetabolic lesions in the caecum and the lung. c Small ulcerous lesion in the cecum with erythematous elevated borders as a manifestation of disseminated histoplasmosis in the gastrointestinal tract. $\mathbf{d}$ Histological examination (40× magnification) of the cecal biopsy revealing numerous large histiocytes (indicated by asterisks) with intracellular fungal elements (indicated by an arrow) in the cytoplasm. 
Doleschal et al.: Disseminated Histoplasmosis: A Challenging Differential Diagnostic Consideration for Suspected Malignant Lesions in the Digestive Tract

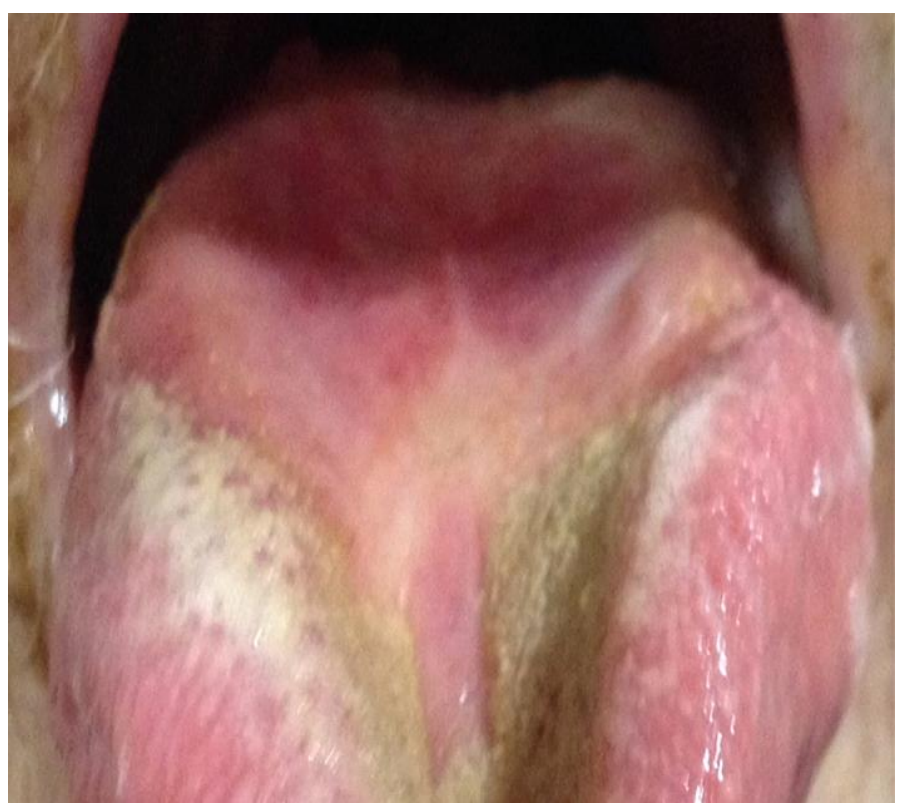

Fig. 3. Follow-up of oral lesions after 6 months of therapy with liposomal amphotericin and itraconazole. 